\title{
INSTITUTIONAL CHANGE
}

Loan Diep (loan.diep.10@ucl.ac.uk) is a Research Assistant and PhD Researcher at UCL Urban Sustainability and Resilience Unit in London and worked as an Urban WASH Researcher at Water \& Sanitation for the Urban Poor; Dr Priti Parikh (priti.parikh@cantab.net) is Associate Professor and Senior Research Fellow with BBOXX and the Royal Academy of Engineering, London; Priti has over 18 years of experience in the WASH sector worldwide; Dr Jonathan Parkinson

(Jonathan.Parkinson@imcworldwide.com) is Associate Director for IMC's Cities and Resilient Development business stream in London, he has a PhD from Imperial College and is co-author of two books focusing on sustainable sanitation and wastewater management; Nii Odai

(nodal@mapleconsult.org) is the Director of MAPLE Consult in Accra, Ghana and works as a development consultant; he has about 30 years of experience in the WASH sector in Ghana

(C) Practical Action Publishing, 2019, www.practicalactionpublishing.org http://dx.doi.org/10.3362/1756-3488.18-00034, ISSN: 0262-8104 (print) 1756-3488 (online)

\section{[CH]Designing stakeholder consultations for institutional change: a case study from Ghana's sanitation sector}

\section{[AU]LOAN DIEP, PRITI PARIKH, JONATHAN PARKINSON, and NII ODAI}

\begin{abstract}
[ABS]This article evaluates the stakeholder consultation process that was undertaken to support decision-making for the new sanitation authority to be established in Ghana. This initiative of creating a specialized authority - currently referred to as the 'National Sanitation Authority' (NSA) emerges from the need to restructure the sector in Ghana, like in many other countries worldwide. From the learning of this study we seek to inform research and practice around the design of stakeholder consultation methods in institutional restructuration contexts. The consultation process gathered views on different aspects of the NSA, including its functions, the administrative level(s) at which it should operate, and its financing. A stakeholder analysis, a series of workshops, and key informant interviews were conducted. A tool was developed to track representativeness of participants involved in the process. Principles of stakeholder engagement developed by the Organisation for Economic Co-operation and Development informed the methodology. This consultation process highlighted the challenges of undertaking sector restructuration where responsibilities are shared between many entities, and with varying levels of interest in the planned reforms. Divergent opinions emerged on the proposed functions of the NSA, some of which represent direct conflicts of interest: for example, setting regulations, developing infrastructure, and financing. Key lessons from the consultation include the need for efficiency when targeting informants through rapid identification of key stakeholders. However, this must be balanced with inclusivity and representativeness, which require continuous tracking of who participates, paired with flexibility to maximize potential for reaching consensus that enables the strengthening of the sanitation sector.
\end{abstract}

[KEY]Keywords: institutional change, sanitation, consultation, stakeholder engagement, Ghana

\section{[A]Objectives and Ghana context}

[B]Consulting stakeholders during institutional change

STAKEHOLDER ENGAGEMENT IS THE PROCESS of bringing together stakeholders, defined as individuals or groups of individuals who are likely to affect or be affected by an intervention, either negatively or positively. It does so in order to align their views and to look for shared and/or mutually compatible 


\section{INSTITUTIONAL CHANGE}

interests (Rietbergen-McCracken and Narayan, 1998; Watt, 2008). By giving the opportunity to those consulted to influence decisions, stakeholder engagement is theoretically recognized as a characteristic of inclusive governance (OECD, 2015). Stakeholder consultation is a type of stakeholder engagement that serves the purpose of moving beyond simple communication. It also recognizes the opportunity of making stakeholders supportive of the proposed change if their concerns are voiced and addressed at relevant stages of the decision-making process.

In a context of institutional change, a consultation would seek to reach out to stakeholders whose experience-sharing can provide valuable inputs to improve programmes, policies, and service delivery. It can also be a means of improving relationships within and between various governance levels, both as a process by bringing individuals or groups of individuals together during discussions, and as an outcome if the institutional change seeks to modify or build on particular dynamics that occur within the existing institutional framework. Nonetheless, while stakeholders can play a significant role in supporting a change during a decision-making process, they can also attempt to block it or divert it by opposing efforts in order to serve their own aims (OECD, 2015).

This article presents and evaluates the methodology which was designed and applied for stakeholder consultation in the context of Ghana's restructuration of its sanitation sector. It seeks to contribute to the literature on stakeholder engagement, and particularly those which focus on consultation processes with governmental institutions (e.g. Rowe and Frewer, 2004; IFC, 2007; NICE, 2012; Department of Public Expenditure and Reform, 2016). It does so in recognition that only a small number of studies have proposed guidelines for the water, sanitation, and hygiene (WASH) sector, or such literature would tend to focus on water (see for example Esogbue and Ahipo, 1982; Seppälä, 2002; Akhmouch and Clavreul, 2016), and rarely in an institutional restructuration context.

As such, the present paper seeks to initiate discussions among both academics and practitioners developing consultation methods for institutional change in the sanitation sector. Ultimately, our goal is to inform decision-making in similar country contexts where a national sanitation authority could be planned for establishment in the future.

\section{$[B]$ The need to restructure Ghana's sanitation sector}

Institutional arrangements, regulations, policies, and bylaws are some of the factors that determine the enabling environment which influences the governance of sanitation services. In Ghana, the sanitation sector is characterized by a complex legal, policy, and strategy framework. Numerous institutions currently work in sanitation in Ghana, with mandates split across different governmental bodies. Box 1 provides an overview of the current institutional structure, while a more comprehensive mapping was undertaken as part of the consultation process (presented in the section headed 'Mapping actors, governance structures, and relationships', below). Several national development plans, such as the 2018-2021 Medium-Term Development Plan and the 2018-2057 Long-Term National Development Plan, include strategies for sanitation. Nevertheless, the country's sanitation frameworks are yet to be fully implemented, and this is reflected in current gaps in sanitation services.

\section{[CAP]Box 1 Overview of stakeholders involved in sanitation in Ghana}

The Ministry of Sanitation and Water Resources (MSWR) formulates and develops policy guidelines, strategies, and standards through the Environmental Health and Sanitation Directorate (EHSD) and the Community Water and Sanitation Agency (CWSA). Regulatory functions are shared between the National Development Planning Commission (NDPC), EHSD, the Environmental Protection Agency (EPA) under the oversight of the Ministry of Environment, Science, Technology and Innovation (MESTI), and the Metropolitan, Municipal and District Assemblies (MMDAs) (who operate under the 


\section{INSTITUTIONAL CHANGE}

Ministry of Local Government and Rural Development, MLGRD). Within the MLGRD, the Office of the Head of Local Government Service (OHLGS) is responsible for ensuring local authorities are staffed with qualified personnel and for providing the capacity building required to deliver their mandates.

There are 16 regions and 260 districts in Ghana in 2019. MMDAs are the decentralized entities responsible for the implementation of national sanitation strategies and policies. Most MMDAs have Environmental Health Sanitation Units (EHSUs) that, among others, conduct monitoring activities. Some MMDAs have undertaken the role of service providers themselves, although the institutional policies state that services should be delegated to private operators. Many MMDAs involved in service provision deal with the construction and management of public toilets. Some MMDAs provide emptying, transport, and treatment services where treatment facilities are available, as in Accra and Kumasi, although this is rare.

The international development community (donors and international NGOs) is largely active in Ghana's sanitation sector. NGOs involved in WASH have formed a member organization, the Coalition of NGOs in Water and Sanitation (CONIWAS). CONIWAS has nearly 100 members. In line with Ghana's national policy for public-private partnerships (adopted in 2011), programmes have given prominence to private sector participation. Donor funding for urban sanitation has increased in recent years but is likely to decrease since Ghana is now considered to be a lower middle-income country according to the World Bank classification.

The private sector, including artisans, masons, private toilet emptiers or public toilet managers, play an important role in local level sanitation, reflecting the increasing participation of the private sector in sanitation service provision. However, this remains limited due to poor engagement and support from governmental institutions and the delivery of sanitation services remains limited on the ground, particularly in low-income areas. For example, there are over 100 privately operated vacuum trucks registered with Accra and Kumasi Municipal Assemblies, but services are not reaching the poor as trucks can rarely access dense low-income areas.

Ghana failed to achieve its Millennium Development Goal (MDG) target, with only 14 per cent improved sanitation coverage and about 19 per cent of the population still defecating in the open. In 2015, it was estimated that 73 per cent of the urban population was relying on shared sanitation facilities (compound and public toilets). Open defecation was practised by 8 per cent of urban dwellers and only 19 per cent had household facilities (WHO/UNICEF, 2017). Only three cities have a sewerage network: Accra, Kumasi, and Tema. Faecal sludge management (FSM) services are developing, but treatment services remain inadequate (Murray and Drechsel, 2011).

Ghana loses approximately GH\$420 m due to poor sanitation each year (equivalent to about US\$290 $m$ in 2012) (WSP, 2012). In 2014, the country committed to allocate 0.5 per cent of its GDP per annum in public funds to sanitation, which corresponds to the equivalent of at least $\$ 150 \mathrm{~m}$ per annum. Domestic public expenditure (excluding household) would have amounted to only GHథ49 $\mathrm{m}(\$ 11.3 \mathrm{~m})$ in 2014 according to some estimations made with the TrackFin finance tracker developed by the World Health Organization (WHO) (Esseku, 2016; Mansour and Esseku, 2017). Therefore, despite the existence of multiple public authorities dedicated to sanitation, the inefficiency of regulatory systems, the weak sanitation market, and the poor investment and co-ordination of existing funds have formed barriers to sector improvement.

The current government that was formed in 2017 and inaugurated the Ministry of Sanitation and Water Resources (MSWR) has initiated an investigation process to establish a national entity dedicated to sanitation. This entity is currently referred to as the National Sanitation Authority (the 


\section{INSTITUTIONAL CHANGE}

NSA) and would help to enact strategic prioritization of sanitation issues in the country. It seeks to address the fragmentation of responsibilities in the sector by bridging the current institutional divide, giving more concentrated attention to sanitation, and improving the activation of policies and strategies on the ground, where low access to safely managed and equitable sanitation services by the population remains a predominant issue.

Other countries including Burkina Faso, Senegal, and Côte d'Ivoire have a national entity dedicated to sanitation. Some of these have been established as asset holders and service providers, and stimulated strong progress in urban sanitation where it was most needed. In Ghana, the rationale behind the establishment of a new entity relates to the failure of the sanitation sector to tackle issues from the national through to the grassroots levels, principally in relation to policy and strategy implementation. A single governmental body dedicated to sanitation is also considered as a way to resolve issues related to duplication and lack of co-ordination among actors and poorly defined legal instruments governing sanitation-related issues. Various models have therefore started to be explored by the Government of Ghana regarding the possible status of the NSA, its functions, and its position within the existing institutional framework.

\section{[A]Methodology}

The MSWR of Ghana invited consultants to aid decision-making around the establishment of the new institutional entity dedicated to sanitation. A two-partner consortium was formed and is composed of WASH researchers and policy translation experts. The consultation process was designed and managed by this team of which several members are the authors of the present article.

\section{[B]Framework}

In the context of Ghana where sanitation responsibilities are fragmented among various entities at multiple governance levels, understanding, assessing, and integrating the range of needs and priorities (i.e. the stakes) of all stakeholders can be a significant challenge. Therefore, the design methodology has focused on balancing inclusivity with efficiency in order to reach a maximum of key groups using the resources and time available to the team.

Building on this purpose, the project's methodology follows the principles on stakeholder engagement of the Organisation for Economic Co-operation and Development (OECD, 2015). Although they focus on the water sector, the policy guidelines of which these principles are part were identified as the most relevant literature to inform the present methodology. It recognizes that all levels of governance have a role to play in establishing an enabling environment for impactful stakeholder engagement. The OECD's set of principles results from comprehensive and detailed work conducted specifically on stakeholder engagement. It was built on empirical data collected from 69 case studies and 215 individuals.

As represented in Table 1, the OECD argues that stakeholder engagement must include six important conditions. Equity enhances the need for engagement opportunities that ensure diverse ideas and opinions are taken into account and are representative of the stakeholder group. Transparency encourages openness about the process of identifying stakeholders, engaging with them, and clearly communicating the goals pursued through the engagement process. Accountability in principle 2 emphasizes the fact that the ideas shared by the stakeholders consulted are actually taken into account. This ensures the legitimacy of decision-making processes and their outcomes. On capacity and information, the guide highlights the need to secure the necessary resources to set up and sustain 


\section{INSTITUTIONAL CHANGE}

appropriate communication channels, whether this is through the creation of an online platform or the organization of a meeting.

On efficiency and effectiveness (the first one referring to the ratio of productivity to effort, and the second one referring to the degree of success in achieving the result pursued), Principle 4 in the OECD guidelines points out that lack of time may be the most common constraint of engagement processes. This is because the engagement process requires time to develop, to be revisited if necessary, while time is also required to build trust with the participants. Principle 5 calls for a process that is aligned coherently and holistically with the existing structure which is engaged with. This means considering place-based needs, as well as adapting to contextual daily practices, professional skills, and the decision-making culture. Finally, adaptiveness also seeks flexibility in the engagement process in order to adapt to changing circumstances, learn from failure, and therefore bounce back accordingly.

\section{[CAP]Table 1 Principles for stakeholder engagement in water governance}

\begin{tabular}{ll}
\hline Inclusiveness and equity & $\begin{array}{l}\text { Principle 1: Map all stakeholders who have a stake in the outcome or that } \\
\text { are likely to be affected, as well as their responsibility, core motivations, } \\
\text { and interactions }\end{array}$ \\
$\begin{array}{ll}\text { Clarity of goals, transparency, } \\
\text { and accountability }\end{array}$ \\
$\begin{array}{l}\text { Principle 2: Define the ultimate line of decision-making, the objectives of } \\
\text { stakeholder engagement, and the expected use of inputs }\end{array}$ \\
Principle 3: Allocate proper financial and human resources and share \\
needed information for result-oriented stakeholder engagement
\end{tabular}

\section{[S]Source: OECD, 2015}

[B]Methods

The set of methods and tools was designed in a way that follows the six OECD principles. The consultation involved engagement with formal institutions, and mostly with governmental bodies and NGOs. Discussions on the development of the NSA were only at an early stage, so consultation with these groups was a priority. However, this recognized that achievement towards the principle of inclusiveness and equity could already be limited.

[C]Stakeholder mapping. Stakeholder mapping is the practice of representing who is responsible for what and at which level. Undertaken at the initial stage of a consultation process, it can help preidentify redundancies and gaps in institutional frameworks that affect policy coherence and sector performance. It also helps in the analysis of interactions between actors at different governance levels. Different types of mapping can be conducted. For this project, two types of mapping were done. Institutional mapping by governance level was first undertaken. Stakeholders were then mapped according to their functions (policy-making, regulation, etc.) (Aligica, 2006; Medilanski et al., 2007; Walker et al., 2008).

[C]Interest-influence matrix. Also part of stakeholder analysis methods, interest-influence matrices are other types of maps that can help represent power dynamics in an existing structure. They help identify stakeholders with a high level of influence and impact. They also help identify what actors are typically excluded from decision-making and planning, and whose engagement should therefore be 


\section{INSTITUTIONAL CHANGE}

actively sought in the consultation. They complement the other maps that show dynamics in the Ghanaian sanitation sector based on policies, with a map that shows dynamics based on perceptions. For this project, the mapping (see Figure 2) was conducted by the Ghana-based part of the team which conducted the consultation, based on their knowledge of the sector (Newcombe, 2003; Polonsky and Scott, 2005).

[C]Workshops. Workshops may be designed in multiple ways in a consultation, depending on the objectives sought. In the case of this consultation, they served the dual purpose of increasing awareness around the range of possible options regarding the NSA's establishment, and triggering group discussions that would explore its scope further and ultimately reach consensus. Two workshops were conducted to brainstorm on the need for a new institutional entity dedicated to sanitation, to present and discuss various international institutional sanitation models, to identify participants' expectations regarding the new entity, and for the research team to present final results (Rietbergen-McCracken and Narayan, 1998; Gregory et al., 2003; Yang et al., 2011).

[C]Tracking tool for gap analysis in participation. In general terms, this gap analysis seeks to identify potential inconsistencies between objectives as set at the beginning of a consultation, and what is actually being conducted during the process. For this consultation, a simple tracking tool was used to keep a record of participants and their level of engagement in the consultation. The tracker listed names and profiles of participating organizations. This was then cross-compared with the list of stakeholders identified during the stakeholder analysis. This was developed for multiple purposes, including to ensure stakeholders from each sanitation governance level had participated in the workshops. This comparison was conducted throughout the entire consultation as it progressed (Rowe and Frewer, 2004; OECD, 2015).

[C]Interviews. A series of interviews was designed when several stages of the consultation had already been completed in order to explore certain aspects of the NSA discussions more deeply and engage further with particular stakeholders including the MSWR. They sought to triangulate information and to include additional views regarding the establishment of the NSA for potential alignment with views and opinions already collected during the workshops. These interviews aimed to reach some stakeholders who did not attend the workshops, including several MMDAs who had been identified as important stakeholders for the consultation (IFC, 2007; Yang et al., 2011).

\section{[A]Consultation process and results}

\section{[B]Mapping actors, governance structures, and relationships}

The stakeholder mapping exercise consisted of representing Ghana's institutional landscape in the post-2016 general election context, which included the creation of the MSWR, under which the NSA is planned to function. It has focused on actors who play a role in sanitation, and particularly attempted to detail those who would potentially have direct interactions with the NSA. This exercise was supported by first-hand knowledge as well as desk-based research, including policy analysis.

Mapping according to governance level is represented in Figure 1. It considered national, district, local, and international levels. It was decided that the regional level would not be represented because it currently largely operates as a conduit from the national through to the local level. The map emphasizes the prominence of actors involved in or affected by the sanitation sector in Ghana, and the associated complex structure of interactions among them.

[CAP]Figure 1 Stakeholders of Ghana's sanitation sector concerned with the establishment of the NSA, by governance level 


\section{INSTITUTIONAL CHANGE}

\section{[S]Source: authors}

Table 2 shows key stakeholders by function in the sector, considering the latest changes following the adoption of recent reforms. This highlighted additional gaps, overlaps, and duplication of responsibilities.

[CAP]Table 2 Key actors intervening in Ghana's sanitation sector by institutional function

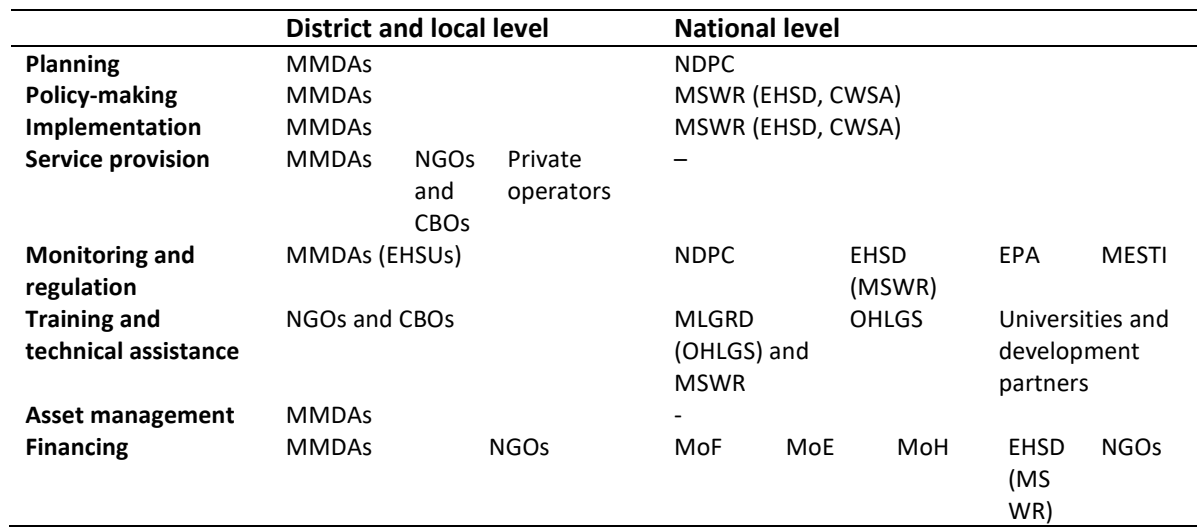

[NOTE]Note: at the regional level, most stakeholders engage in monitoring and evaluation at the district and local levels.

[S]Source: based on Mansour and Esseku, 2017

Both mapping exercises (Figure 1 and Table 2) highlighted the importance of the institutional role of the MMDAs at the local level and the way they link to other institutions and organizations, particularly the MSWR and MLGRD at national level. However, the level of detail chosen for these types of mapping is context-dependent, and is defined according to the scope of the exercise. For example, while the governance-level map focuses on national entities, the Environmental Health Sanitation Units (EHSUs) were added because of their central role in enforcing regulations and in promoting sanitation activities which is highly relevant in the context of the NSA. Yet, the choice was made not to detail all of the MMDAs' sub-entities, including committees that operate under their authority such as the works sub-committees whose function covers sanitation infrastructure at a minimum level (Friedrich-Ebert-Stiftung Ghana, 2016).

The stakeholder analysis also helped with the identification of governance gaps and inconsistencies across stakeholders' responsibilities at multiple levels. The private sector (including small and medium-sized enterprises) remains an important actor, including for service delivery on the ground. However, it has limited institutional responsibilities despite policies encouraging MMDAs to delegate service provision. Artisans, masons, and vacuum track operators play key roles in service provision, but the current legal and regulatory framework does not yet provide them with a contractual environment that is favourable enough to their integration in the institutional landscape.

Besides, research conducted to undertake the mapping helped identify potential conflicts among institutions and organizations. The international community has been recognized as playing an influential role, for example since an important part of financing in sanitation comes from donor funding. However, this has helped to highlight that donors are sometimes testing multiple and 


\section{INSTITUTIONAL CHANGE}

sometimes conflicting approaches, for example in relation to investment models or capacity-building strategies implemented with different actors at different levels.

On the consultation process itself, the stakeholder maps have also helped monitor the actors and groups of actors who have been engaged with. The categorization of stakeholders by governance level was re-used to design the tracking tool used for gap analysis to answer questions such as 'what organization was consulted?' And 'what governance level does it represent?'

\section{$[\mathrm{B}]$ Interest-influence analysis}

The interest-influence analysis sought to complement the stakeholder mapping by representing dynamics and power relations in Ghana's sanitation sector (Figure 2). It was conducted by Maple Consult, the Ghanaian-based partners of this consultation project, independently from the rest of the consulting team. The mapping responded to the question 'What are the organization/institutions and their level of influence and interest in the establishment of the NSA?' A scoring system from 1 to 5 (where 1 is low and 5 is high) was used for this purpose as represented in Figure 2. The figure shows the range of medium-to-high influence organizations invited to participate in the consultation.

Several stakeholders that were not identified during the stakeholder mapping were considered for the interest/influence matrix. This includes the State Enterprises Commission and the Public Services Commission which has to recognition of the need for an authority to be established before the government can lend support to it especially with regard to human resources. Although indirectly represented through CONIWAS, international organizations such as UNICEF were indicated in the matrix as one of the key funding organizations for the sanitation sector in Ghana. The Ministry of Gender, Children and Social Protection, Ghana Water Company Ltd, and Land-Use \& Spatial Planning Authority were other relevant organizations included in the interest-influence mapping.

Differences between this mapping process and the previous ones presented above are important to highlight differences between roles and functions as defined in policies and frameworks with actual power executed on the ground. They also emphasize the way perceptions that vary across those conducting stakeholder mapping exercises may significantly affect results. For instance, WSUP's influence and impact regarding the establishment of the NSA was highlighted here because of its advocacy role, although it was not specifically represented on the stakeholder maps. In contrast, government entities such as EHSD were considered as having a central role in the sector in both stakeholder maps, but are not represented in the matrix.

[CAP]Figure 2 Stakeholder analysis on interest in and influence on NSA establishment according to Maple Consult

\section{[B]Workshops}

An inception meeting was first organized with MSWR representatives. This meeting served the dual purpose of presenting the consultation process and its modalities to the MSWR, as well as MSWR sharing its views on the opportunities of and the barriers to the NSA's establishment. The latter helped establish the scope of discussions undertaken during the further steps of the consultation.

The workshops centred discussions on the role of the NSA within the existing institutional structure, and particularly 1 ) the rationale for the NSA's establishment; 2 ) opinions on the focus and scope of the NSA; 3) levels of operations; 4) interactions with other key stakeholders; 5) funding; and 6) staffing. The first workshop focused on discussing existing international institutional models of sanitation sectors, and to reflect on what that meant for the Ghana context. Models of six countries (Burkina Faso, India, Senegal, Kenya, Zambia, and Uganda) were presented for discussions and increasing

Commented [G1]: In Figure 2, WHO is mentioned in the notes but does not seem to be on the figure itself. Please check 


\section{INSTITUTIONAL CHANGE}

awareness around advantages and disadvantages of these. The second workshop focused on discussing specific functions and roles of the NSA in Ghana.

The first workshop was attended by 21 stakeholders from 19 organizations. Of the 21 , there were six participants from governmental departments/ministries, two from the private sector, and the remainder from non-governmental/donor agencies; 16 participants were male and five were female. The second workshop was attended by 44 participants from 18 organizations. Eight participants attended both workshops.

Participants in the first workshop took part in a ranking exercise focused on the NSA's functions. The first ranking exercise required participants to rank from 0 to 5 each of the 14 functions proposed ( 0 being attributed to functions that should not be fulfilled by the NSA, and 5 being attributed by those who felt NSA should undertake them). Results in Figure 3 show a stronger preference for 'monitoring' and 'setting standards'. 'Service provision' and 'project implementation' received the lowest score as most participants viewed this function to be outside the NSA's scope. Figure 4 represents the number of participants who attributed 5 to each function, showing that 16 out of 21 participants attributed a score of 5 to 'monitoring'.

[CAP]Figure 3 Average of scores attributed by workshop participants to each function proposed for the NSA

[CAP]Figure 4 Number of participants attributing a score of 5 to each category $(5=$ the NSA to take on the identified role)

\section{[B] Gap analysis and extended consultation}

A simple consultation tracking tool was developed to keep record of participants (including the organization they represented and their function). This supported the rapid identification of gaps in institutional representation in the consultation process, including the limited participation of local entities such as SMEs, academic institutions, and development organizations closely working with civil society.

A series of interviews with four MMDAs, two schools of hygiene, and eight NGOs were included in the consultation process following gap analyses using the consultation tracking tool. These were conducted outside Accra. In total, 24 interviews with governmental organizations, civil society groups, NGOs, and international NGOs helped in reaching out to further stakeholders who did not take part in the workshops.

\section{[A]Consultation assessment}

This section discusses the extent to which the six principles of the OECD's guidance on stakeholder engagement were met during the consultation process.

\section{[B]Inclusiveness and equity}

The stakeholder analysis, through stakeholder mapping and the influence-interest matrix, was conducted in a way that would ensure that 'all stakeholders' with a direct and indirect stake in the outcome of the NSA's establishment would be identified. The pursuit of this principle led to engagement with a large number of actors whose opinions were integrated in discussions at subsequent stages. Yet, having numerous actors involved does not necessarily ensure representativeness, and, in fact, many groups were poorly represented, including civil society and SMEs at local level. Besides, engagement with a high number of actors increased the risk of affecting efficiency and effectiveness during the consultation process. 


\section{[B]Clarity of goals, transparency, and accountability}

The consultation was designed to ensure continuity in communication with participants, including through the sharing of objectives and outcomes throughout the process. The objectives of the consultation were communicated to workshop participants in advance by co-ordinators, but the questionnaires used to evaluate this demonstrated that this had not been clear for all participants. The final workshop was conducted to follow up on outcomes of previous workshops but was attended by different stakeholders. Therefore, continuity in decision-making was affected.

\section{[B]Capacity and information}

The size of the team conducting the consultation helped ensure that inputs were appropriate to the objectives of the exercises conducted. The initial meeting with the MSWR was key to start engagement with an institutional body central to the establishment of the NSA. Further budget was released to conduct the extended consultation. Nevertheless, as mentioned above, the condensed timescale and scope of the consultation meant that it was not extended to wider groups.

Regarding information, the consultation itself was a means of communicating with stakeholders about the government's intention to establish the NSA. Prior to the consultation, all but two of the stakeholders consulted were aware of ongoing discussions regarding the NSA. All stakeholders considered themselves more engaged in the consultation after the workshop. As captured by the questionnaires, they entered the first workshop with an average perception of being engaged (score of 3.1/5) to feeling highly engaged (score of 4.15/5). For the first workshop, 11 out of 21 participants felt that their perception of the NSA had significantly or partly changed after the workshop. Sharing of information via online platforms for discussions would have also allowed for wider consultation.

\section{[B]Efficiency and effectiveness}

The tracking tool helped identify that many participants in the workshop were occupying junior and/or niche positions, while inputs from individuals with a wide understanding of the sector as a whole could have helped maximize the use of inputs.

Assessing stakeholder engagement throughout the process using the tracking tool helped to decide when and how to engage with particular individuals. For example, as participation and inputs from MSWR were assessed as high priority, holding a preliminary workshop and further interviews with MSWR representatives meant that their inputs could be maximized as opposed to solely inviting them to participate in workshops.

By grouping stakeholders by governance levels (international, national, district, and local), the gap analysis using the tracking tool helped ensure there was representation from each group. However, this also highlighted the difficulty in balancing quality of inputs, comprehensive representation, and a manageable number of participants. In this sense, seeking efficiency and effectiveness might affect inclusivity and input value. Having a further breakdown of groups and/or setting a minimum number of participants per group could have helped manage this balance.

Furthermore, respondents highlighted that key materials, including a draft of the proposed NSA structure, information on proposed models and structure for the NSA, a context analysis, and the presentations used in workshops, would have been helpful to facilitate discussions if shared in advance. The lack of an official document to refer to as the basis for the consultation was identified as a weakness of the process. 


\title{
INSTITUTIONAL CHANGE
}

\begin{abstract}
[B]Institutionalization, structuring, and integration
Various processes were established in order to respect established in-organization decision-making structures. That included formal workshop invitations being sent to the relevant human resources authority or the leader of the different organizations in order for them to decide on the representative who would attend the event. As such, the nature of the participating organization would remain within the organization itself. However, this also increased the risk of the responsibility being delegated to individuals who have little knowledge or experience of the topic being discussed during the event.
\end{abstract}

\section{[B]Adaptiveness}

While there had been no considerable changing circumstances affecting the consultation, the process's flexibility was evidenced by the addition of interviews for wider stakeholder engagement. This was helped by the gap tracking exercise conducted after the first workshop. It highlighted the need to reach additional organizations including local assemblies to ensure national, regional, and local level representation. It also led to the decision to hold small group interviews as opposed to bigger workshops. The flexibility of the consultation in this circumstance therefore helped customize the type and level of engagement with certain stakeholders who had been not involved enough.

\section{[A]Conclusion and recommendations}

As the final stages of the decision-making process for the NSA is still ongoing, a full assessment of the extent to which the consultation has influenced decisions could not be made. However, it appears inevitable that some suggestions from participants will be prioritized over others, since certain proposed functions for the NSA may conflict with each other. For example, the NSA could not simultaneously adopt the role of setting regulations and/or managing some budget of the sector, while also initiating infrastructure development and potentially acting as a service provider at the local level without compromising its institutional independence. While the consultancy was successful in gathering and combining the views of multiple stakeholders, a key lesson is that these diverging views cannot be implemented in their totality as a risk of losing coherence would emerge.

As this study emphasizes the challenge of engaging with a wide range of stakeholders with varying levels of influence in consultations, it also touches upon the introduction of biases introduced throughout the process and thereby influencing results. Stakeholder engagement processes require careful and strategic articulation of the range of perceptions and opinions gathered in order to eventually reach decisions that reflect this variety of views. As such, different stages must be designed to navigate the complexity of collecting feedback from individuals and organizations whose interests might diverge and possibly conflict with each other.

In this particular case of the establishment of the NSA in Ghana, the process showed weaknesses regarding the mobilization of participants because the consultation required individuals with sector experience, but there were factors over which we had limited control. For example, decision-making structures and culture within institutions/groups would determine their participation and internal choice of representative. Individuals who tend to be considered as those with greater experience of the sector, and who may be holding senior positions within such structures, would have limited availability for consultation processes. This is a key factor to consider in consultations as it directly influences results. This further raises the matters of 'who should participate?', 'whose knowledge counts?', 'what constitutes a legitimate stake?', and thereby questions the extent to which a consultation can be inclusive (Reed et al., 2009). 


\section{INSTITUTIONAL CHANGE}

Additionally, the involvement of smaller, local, sometimes informal groups or organizations may be considered further. In this research, the team chose to conduct interviews with groups that were not easily reachable via the formal workshops conducted. However, this compromises consistency in the way inputs are collected. It also poses the question of group dynamics and their influences, and if the participation of these groups in the workshops would have led to different outcomes.

Based on the analysis of the outcomes of the consultation, the authors suggest the following recommendations for organizations conducting consultations in the sanitation sector for similar purposes.

\section{$[B]$ Start by capturing views on the rationale behind institutional change}

Initiating discussions on the problems to address (e.g. 'why do we need the NSA?') aids brainstorming, helps align participants' expectations and steer discussions. Commonly identified problems should subsequently be formulated in a manner that facilitates wider-ranging analysis. Referring back to these underlying problems regularly, and therefore to the consultation's ultimate objectives, helps set the parameters within which to steer the direction of discussions.

\section{[B]Ensure participants' time and inputs are used effectively}

Taking part in workshops is time-consuming and costly, which is the reason why turnout is not always as high as expected. Ensuring the adequate preparation of discussions, communicating clear aims prior to workshops, and targeting the right participants from the beginning are crucial steps to ensure that stakeholder engagement is maximized. This is particularly important to avoid a 'consultation fatigue' that many participants may experience. Guiding discussions with pre-prepared optional 'solutions' to debate (but ensuring they do not restrict debate) can facilitate decision-making. Giving organizations the opportunity to review documents internally and consider key pre-prepared questions before workshops can significantly quicken the pace of consultation, with a representative bringing collective feedback to the workshop.

\section{[B]Quickly identify gaps to ensure stakeholders with high interest but low levels of influence are} included

The purpose of stakeholder mapping and tracking representation is to identify, incorporate the needs of, and draw on the experience of all stakeholders. Individuals with high levels of experience can be consulted in smaller core steering group meetings on a more frequent and in-depth basis. Recognizing those who have lower levels of influence but high interest is also likely to benefit outcomes, giving visibility to issues that might not otherwise be acknowledged. A key recommendation is to target these organizations and engage them as much as possible. Regarding the interest-influence analysis specifically, triangulation is necessary to optimize results. For this study, it would have been relevant to get all institutions to rate all the other institutions according to interest and influence; these assessments could have formed the basis of group work and discussion in a workshop.

\section{[B]Get local bodies actively engaged}

Because of their responsibilities for policy-making, planning, implementation, monitoring, regulation, and even service provision in certain cases, local bodies have a key role to play in sanitation in Ghana. More generally in sanitation sectors worldwide, local governments are well-placed to recognize challenges regarding service provision and demand on the ground, while they also understand the institutional and financial challenges. It is therefore crucial that their needs and opinions are recognized and they are actively involved throughout the consultation.

\section{$[B]$ Engage with civil society and the private sector}




\section{INSTITUTIONAL CHANGE}

In Ghana, there is a dynamic private sector involved at all stages of the sanitation chain, from toilet construction to emptying and treatment. Increasing demand for services and raising awareness of sanitation risks, particularly among low-income citizens, will be key. Early consultation stages regarding the NSA's establishment mainly targeted government representatives and the development community such as international and national NGOs. However, further consultation before any final decisions are made (accompanied by adequately allocated budget) with the private sector and civil society will ensure the process appropriately embraces all key principles of stakeholder engagement, including that of inclusivity.

[FULL OUT]As a final conclusion, we call for further work to be conducted regarding the way consultations manage the balance between the need for efficiency one the one hand, and inclusiveness and representativeness on the other hand. While the OECD guidance is relevant to such research context, it is important to note that it is not enough on its own to design a consultation that fully achieves the principles, but that every methodology deriving from this guidance is contextually designed. We therefore invite further research to be conducted on the way the principles can be used to design methods and monitor the extent to which they are achieved. This will become increasingly relevant as institutions worldwide are revisiting their own national institutional frameworks to better address sanitation challenges and create changes in the sector.

\section{[A]References}

Akhmouch, A. and Clavreul, D. (2016) 'Stakeholder engagement for inclusive water governance: 'practicing what we preach' with the OECD Water Governance Initiative', Water (Switzerland) 8(5): $1-17<$ http://dx.doi.org/10.3390/w8050204>.

Aligica, P.D. (2006) 'Institutional and stakeholder mapping: frameworks for policy analysis and institutional change', Public Organization Review 6(1): 79-90 <http://dx.doi.org/10.1007/s11115006-6833-0>.

Department of Public Expenditure and Reform (2016) Consultation Principles \& Guidance [pdf], Dublin <https://assets.gov.ie/5579/140119163201-9e43dea3f4b14d56a705960cb9354c8b.pdf> [accessed 30 June 2019].

Esogbue, A.O. and Ahipo, Z.M. (1982) 'A fuzzy sets model for measuring the effectiveness of public participation in water resources planning', Journal of the American Water Resources Association 18(3): 451-6 <http://dx.doi.org/10.1111/j.1752-1688.1982.tb00011.x>.

Esseku, H. (2016) TrackFin Initiative: Results from Ghana, 27th Mole Conference [pdf] $<$ https://www.washghana.net/sites/default/files/Mole\%20-

\%20TrackFin\%20Ghana\%20Nov\%2022\%2C\%202016.pdf> [accessed 25 June 2019].

Friedrich-Ebert-Stiftung Ghana (2016) A Guide to District Assemblies in Ghana [pdf], ILGS and Friedrich-Ebert-Stiftung <http://www.fesghana.org/uploads/PDF/DISTRICT ASSEMBLY_2nd Edition.pdf> [accessed 21 February 2019].

Gregory, R., Fischhoff, B., Thorne, S., and Butte, G. (2003) 'A multi-channel stakeholder consultation process for transmission deregulation', Energy Policy 31: 1291-99 < https://doi.org/10.1016/S03014215(02)00188-X>.

IFC (2007) Stakeholder Engagement: A Good Practice Handbook for Companies Doing Business in Emerging Markets [pdf], Washington, DC: IFC <https://www.ifc.org/wps/wcm/connect/938f1a0048855805beacfe6a6515bb18/IFC_StakeholderEn gagement.pdf?MOD=AJPERES> [accessed 25 June 2019]. 


\section{INSTITUTIONAL CHANGE}

Mansour, G. and Esseku, H. (2017) Situation Analysis of the Urban Sanitation Sector in Ghana [pdf], Urban Sanitation Research Initiative Ghana, London

$<$ https://www.wsup.com/content/uploads/2017/09/Situation-analysis-of-the-urban-sanitationsector-in-Ghana.pdf> [accessed 25 June 2019].

Medilanski, E., Chuan, L., Mosler, H-J., Schertenleib, R., and Larsen, T.A. (2007) 'Identifying the institutional decision process to introduce decentralized sanitation in the city of Kunming (China)', Environmental Management 39(5): 648-62 <http://dx.doi.org/10.1007/s00267-005-0321-0>.

Murray, A. and Drechsel, P. (2011) 'Why do some wastewater treatment facilities work when the majority fail? Case study from the sanitation sector in Ghana', Waterlines 30(2): 135-49 <http://dx.doi.org/10.3362/1756-3488.2011.015>.

Newcombe, R. (2003) 'From client to project stakeholders: a stakeholder mapping approach', Construction Management and Economics 21(8): 841-8

<http://dx.doi.org/10.1080/0144619032000072137>.

NICE (2012) The Guidelines Manual: Process and Methods [online], London $<$ http://www.nice.org.uk/aboutnice/howwework/developingniceclinicalguidelines/clinicalguidelined evelopmentmethods/GuidelinesManual2009.jsp> [accessed 25 June 2019].

OECD (2015) Stakeholder Engagement for Inclusive Water Governance, Paris: OECD <http://dx.doi.org/10.1787/9789264231122-en> [accessed 25 June 2019].

Polonsky, M.J. and Scott, D. (2005) 'Empirical examination of the stakeholder strategy matrix', European Journal of Marketing 39(9-10): 1199-215 <http://dx.doi.org/10.1675/15244695(2008)31>.

Reed, M.S., Graves, A., Dandy, N., Posthumus, H., Hubacek, K., Morris, J., Prell, C., Quinn, C.H., and Stronger, L.C. (2009) 'Who's in and why? A typology of stakeholder analysis methods for natural resource management', Journal of Environmental Management 90(5): 1933-49 <http://dx.doi.org/10.1016/j.jenvman.2009.01.001>.

Rietbergen-McCracken, J. and Narayan, D. (1998) Participation and Social Assessment: Tools and Techniques, Washington, DC: World Bank.

Rowe, G. and Frewer, L.J. (2004) 'Evaluating public-participation exercises: a research agenda', Science Technology and Human Values 29(4): 512-57 <http://dx.doi.org/10.1177/0162243903259197>.

Seppälä, O.T. (2002) 'Effective water and sanitation policy reform implementation: need for systemic approach and stakeholder participation', Water Policy 4(4): 367-88 <http://dx.doi.org/10.1016/S1366-7017(02)00036-3>.

Walker, D.H.T., Bourne, L.M., and Shelley, A. (2008) 'Influence, stakeholder mapping and visualization', Construction Management and Economics 26(6): 645-58 <http://dx.doi.org/10.1080/01446190701882390>

Watt, E. (2008) A Framework for Evaluating Institutional Stakeholder Engagement at the Ministry of Health Services, Victoria, BC: University of Victoria <https://dspace.library.uvic.ca/bitstream/handle/1828/1222/watt_eben.pdf?sequence=1> [accessed 19 February 2019].

WHO/UNICEF (2017) 'JMP Data: Ghana' [online] <https://washdata.org/data/household\#!/> [accessed 30 June 2019].

WSP (2012) Ghana Loses GHC420 Million Annually due to Poor Sanitation, Washington, DC: WSP. 
L. DIEP ET AL.

DESIGNING STAKEHOLDER CONSULTATIONS FOR

\section{INSTITUTIONAL CHANGE}

Yang, J., Shen, P.Q., Bourne, L., Ho, C.M.F., and Xue, X. (2011) 'A typology of operational approaches for stakeholder analysis and engagement', Construction Management and Economics 29(2): 145-62 <http://dx.doi.org/10.1080/01446193.2010.521759>. 